\title{
Linearized Riesz Transform and Quasi-Monogenic Shearlets
}

\author{
S. Häuser*, B. Heise ${ }^{\dagger}$ and G. Steidl*
}

July 29, 2013

\begin{abstract}
The only quadrature operator of order two on $L_{2}\left(\mathbb{R}^{2}\right)$ which covaries with orthogonal transforms, in particular rotations is (up to the sign) the Riesz transform. This property was used for the construction of monogenic wavelets and curvelets. Recently, shearlets were applied for various signal processing tasks. Unfortunately, the Riesz transform does not correspond with the shear operation. In this paper we propose a novel quadrature operator called linearized Riesz transform which is related to the shear operator. We prove properties of this transform and analyze its performance versus the usual Riesz transform numerically. Furthermore, we demonstrate the relation between the corresponding optical filters. Based on the linearized Riesz transform we introduce finite discrete quasimonogenic shearlets and prove that they form a tight frame. Numerical experiments show the good fit of the directional information given by the shearlets and the orientation obtained from the quasi-monogenic shearlet coefficients. Finally we provide experiments on the directional analysis of textures using our quasi-monogenic shearlets.
\end{abstract}

Keywords: Shearlets, Riesz Transform, monogenic signals, local orientation, instantaneuos phase

\section{Introduction}

Based on the Riesz transform monogenic signals were introduced in image processing by Felsberg and Sommer [7] and in optics by Larkin et al. [18]. The Riesz transform of a twodimensional signal provides information about the amplitude, the instantaneous phase and the local orientation (of the phase) of the signal. Therefore it contains, in contrast to, e.g., the directional Hilbert transform where the desired direction has to be addressed in advance, an 'automatic' orientation component. The Riesz transform can replace the (smoothed) gradient in structure tensors as those of Förstner and Gülch [8] to make them more robust, see, e.g., $[16]$.

In practice the Riesz transform is often applied on some bandpass filtered version of the signal. This idea was adopted in the construction of monogenic wavelets in $[15,27,28]$, where the first paper is based on Meyer wavelets while the other ones focus on spline wavelets. The monogenic wavelet coefficients of the signal provide amplitude, phase and orientation information on multiscales now. Monogenic wavelet transforms were applied for tensor based estimation of orientations, demodulation of interferograms and the reconstruction of holograms in $[27,28]$, and for brightness equilibration and parameter free descreening in [15].

\footnotetext{
${ }^{*}$ University of Kaiserslautern, Dept. of Mathematics, Kaiserslautern, Germany

${ }^{\dagger}$ J. Kepler University Linz, Ch. Doppler Laboratory for Microscopic and Spectroscopic Material Characterization, Austria
} 

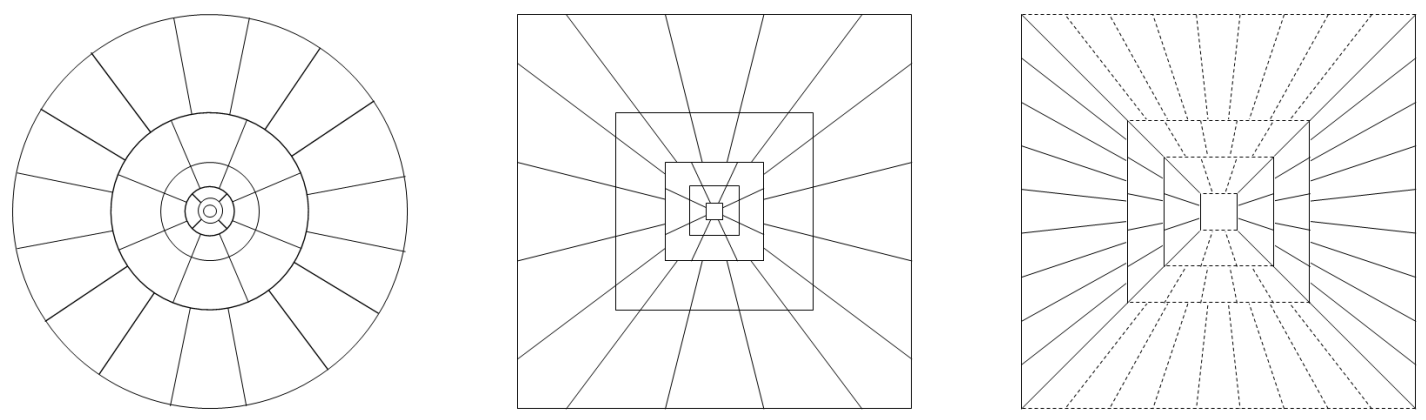

Figure 1: (left to right) Tilings of the frequency plane by the support of the (i) second generation curvelets [3] $\left(4 \cdot 2^{\lceil j / 2\rceil}\right.$ wedges at scale $\left.2^{-j}\right)$, (ii) discrete curvelets [4] with pseudopolar support, (iii) cone-adapted shearlets [11, 13].

Recently the monogenic curvelet transform was introduced by Storath [26]. He proved that this transform behaves at very fine scales like the usual curvelet transform and at coarse scales like the monogenic wavelet transform. Since the Riesz transform covaries with rotations, the monogenic curvelet amplitude is invariant to the rotation group operation of the curvelet transform. This is useful as long as the curvelet transform is defined with respect to curvelets which supports tile the frequency plane into concentric circle segments as originally proposed for second generation curvelets by Candés and Donoho in [3], see Fig. 1 left. However, in implementations Cartesian arrays are prefered over the polar tiling. The fast discrete curvelet transform [4] replaces the polar tiling of the frequency plane by Cartesian coronae based on concentric squares (instead of circles) and shears [4], see Fig. 1 middle. Of course, these curvelets cannot be obtained by rotating a mother curvelet on the respective scale. For a review on curvelets see [20].

An approach which takes Cartesian arrays from the beginning into account are shearlets which were introduced in [19]. Instead of rotations the group of shear matrices is involved into the construction. In this paper we focus on the cone adapted version of shearlets given in [11]. The tiling of the frequency plane by the support of these shearlets is shown in Fig. 1 right. Shearlets have the same nice approximation properties as curvelets and have found applications, e.g., for edge detection in $[12,17]$. Moreover, shearlets as wavelets are directly related with square integrable group representations [5] and function spaces [6]. Of course the Riesz transform does not covaries with the shear operation.

The aim of this paper is twofold. First, we introduce a linearized Riesz transform which better fits to the cone-like frequency tiling in Fig. 1 middle/right. This transform is a quadrature operator related to the shear operation in the sense that, roughly speaking, the linearized Riesz transform of a sheared function equals the sheared linearized Riesz transform of the function up to a rotation by the shear factor. We demonstrate properties of the linearized Riesz transform both from the mathematical implementation and the optical filter point of view. Second we introduce quasi-monogenic shearlets as counterpart to monogenic curvelets by applying the linearized Riesz transform instead of the usual Riesz transform.

The paper is organized as follows: In Section 2 we review quadrature operators and consider 
a special one, namely the Riesz transform. We introduce the linearized Riesz transform and corresponding quasi-monogenic signals in Section 3. We prove some relevant properties of our linearized version and compare the transform numerically with the ordinary Riesz transform. The relation between the linearized and the ordinary Riesz transform is also considered for the corresponding optical filters. Section 4 deals with quasi-monogenic shearlets. Finally, various numerical examples on the analysis of directions in textures are given in Section 5. The local orientation of the quasi-monogenic shearlet coefficients gives directly the orientation of the phase in the texture features.

\section{Quadrature Operators and Riesz Transform}

In this section we briefly review the notation of quadrature operators. For more information on these topics we refer to $[9,15,25]$. In the next section we will see that our linearized Riesz transform is as the Riesz transform itself a quadrature operator. Let $\mathbb{K} \in\{\mathbb{R}, \mathbb{C}\}$ and let $\left\{\boldsymbol{e}_{0}, \ldots, \boldsymbol{e}_{m}\right\}$ denote the standard orthonormal basis of $\mathbb{K}^{1+m}$. We equip $\mathbb{K}^{1+m}$ with the vector multiplication

$$
\boldsymbol{e}_{\mu} \boldsymbol{e}_{\mu}=-\boldsymbol{e}_{0}, \quad \boldsymbol{e}_{\nu} \boldsymbol{e}_{\mu}=-\boldsymbol{e}_{\mu} \boldsymbol{e}_{\nu}, \quad \mu \neq \nu, \nu, \mu=1, \ldots, m .
$$

The elements of $\mathbb{K}^{1+m}$, called hypercomplex numbers, can be represented as

$$
v=v_{0}+\sum_{\mu=1}^{m} \boldsymbol{e}_{\mu} v_{\mu},
$$

where we write as usual 1 for the multiplicative identity $\boldsymbol{e}_{0}$. In analogy to complex numbers, the $\mathbf{e}_{\mathbf{0}}$ component is called real part and the other components imaginary part of $v$. The closure of $\mathbb{K}^{1+m}$ under the above multiplication is the Clifford algebra $\mathbb{K}_{m}$ of dimension $2^{m}$. In particular, the multiplicative closure of $\mathbb{R}^{1+1}$ are the complex numbers and of $\mathbb{R}^{1+2}$ are the quaternions. In the following, we only consider powers of two of elements in $\mathbb{K}^{1+m}$ which are again in $\mathbb{K}^{1+m}$. We define the vector-valued bilinear form $(\cdot, \cdot)$ for $F=\left(f_{\mu}\right)_{\mu=0}^{m} \in$ $L_{2}\left(\mathbb{R}^{n}, \mathbb{K}^{1+m}\right)$ and $g \in L_{2}\left(\mathbb{R}^{n}, \mathbb{K}\right)$ by

$$
(F, g):=\sum_{\mu=0}^{m} \boldsymbol{e}_{\mu}\left\langle f_{\mu}, g\right\rangle,
$$

where $\langle\cdot, \cdot\rangle$ denotes the $L_{2}$ inner product. Further we use the Fourier transform $\mathcal{F}: L_{2}\left(\mathbb{R}^{n}, \mathbb{C}\right) \rightarrow$ $L_{2}\left(\mathbb{R}^{n}, \mathbb{C}\right)$ given by

$$
\mathcal{F} f(\omega)=\hat{f}(\omega):=\int_{\mathbb{R}^{n}} f(t) e^{-2 \pi i\langle\omega, t\rangle} d t .
$$

We will frequently use that for a linear, invertible operator $A \in \mathbb{R}^{n, n}$ the relation

$$
\left(f\left(A^{-1} \cdot\right)\right)^{\wedge}(\omega)=|\operatorname{det} A| \hat{f}\left(A^{\mathrm{T}} \omega\right)
$$

holds true. For $F \in L_{2}\left(\mathbb{R}^{n}, \mathbb{K}^{1+m}\right)$ we apply the Fourier transform componentwise

$$
\hat{F}(\omega):=\sum_{\mu=0}^{m} \boldsymbol{e}_{\mu} \hat{f}_{\mu} .
$$


For an $m$-tuple of linearly independent, bounded, linear operators $Q_{1}, \ldots, Q_{m}: L_{2}\left(\mathbb{R}^{n}, \mathbb{R}\right) \rightarrow$ $L_{2}\left(\mathbb{R}^{n}, \mathbb{R}\right)$ we call

$$
\mathcal{Q}:=\sum_{\mu=1}^{m} \mathbf{e}_{\mu} Q_{\mu}
$$

a quadrature operator of order $m$ if the following conditions are fulfilled:

1. $\mathcal{Q}$ is invariant under translation,

2. $\mathcal{Q}$ is invariant under positive dilations,

3. $\mathcal{Q}$ is self-inverting, i.e., $\mathcal{Q}^{2}=\mathrm{id}$,

4. $Q_{\mu}$ is anti-selfadjoint, i.e., $Q_{\mu}^{*}=-Q_{\mu}, \mu=1, \ldots, m$.

For a quadrature operator $\mathcal{Q}$ we define the $\mathcal{Q}$-complex signal operator $\mathcal{Q}^{\prime}$ on $L_{2}\left(\mathbb{R}^{n}, \mathbb{R}\right)$ by

$$
\mathcal{Q}^{\prime} f:=f+\sum_{\mu=1}^{m} \mathbf{e}_{\mu} Q_{\mu} f
$$

For $f \in L_{2}\left(\mathbb{R}^{n}, \mathbb{R}\right)$ the $\mathcal{Q}$-amplitude is determined by

$$
\left|\mathcal{Q}^{\prime} f(x)\right|:=\sqrt{|f(x)|^{2}+\left|Q_{1} f(x)\right|^{2}+\ldots+\left|Q_{m} f(x)\right|^{2}} .
$$

The angle $\xi \in[0, \pi]$ defined by

$$
\xi\left(\mathcal{Q}^{\prime} f(x)\right):=\arccos \frac{f(x)}{\left|\mathcal{Q}^{\prime} f(x)\right|}, \quad\left|\mathcal{Q}^{\prime} f(x)\right| \neq 0
$$

is the phase of $\mathcal{Q}^{\prime} f$.

Two important quadrature operators are the (partial) Hilbert transform in direction $\omega_{0} \in$ $\mathbb{R}^{n} \backslash\{0\}$ defined for $f \in L_{2}\left(\mathbb{R}^{n}, \mathbb{R}\right)$ by

$$
\widehat{\mathcal{H}_{\omega_{0}} f}(\omega):=-i \operatorname{sgn}\left(\left\langle\omega, \omega_{0}\right\rangle\right) \hat{f}(\omega) .
$$

and the Riesz transform defined componentwise by

$$
\widehat{\mathcal{R}_{\mu} f}(\omega):=-i \frac{\omega_{\mu}}{|\omega|} \hat{f}(\omega), \quad \mu=1, \ldots, n .
$$

In the spatial domain $\mathcal{R}: L_{2}\left(\mathbb{R}^{2}\right) \rightarrow\left(L_{2}\left(\mathbb{R}^{2}\right)\right)^{2}$ is the convolution with the distribution $\frac{\Gamma\left(\frac{n+1}{2}\right)}{\pi^{n+1 / 2}} \mathrm{PV} \frac{t}{|t|^{n+1}}$.

For $n=1$, we know that $\boldsymbol{e}_{1} \mathcal{H}$ with the Hilbert transform defined for $f \in L_{2}\left(\mathbb{R}^{n}, \mathbb{R}\right)$ by $\mathcal{H} f(\omega):=-i \operatorname{sgn}(\omega) \hat{f}(\omega)$ and its negative variant $-\boldsymbol{e}_{1} \mathcal{H}$ are the only quadrature operators of order $m=1$. The corresponding $\mathcal{H}$-complex signal $f+\boldsymbol{e}_{1} \mathcal{H} f$ of $f \in L_{2}\left(\mathbb{R}^{n}, \mathbb{R}\right)$ is known as analytic signal of $f$.

For $n>1$, the partial Hilbert transform is a quadrature operator of order $m=1$. The Riesz transform is (up to the sign) the only quadrature operator of order $n$ that covaries with linear, 
orthogonal transforms. In other words, if $U \in \mathbb{R}^{n, n}$ is an orthogonal matrix, i.e., $U^{-1}=U^{\mathrm{T}}$, then

$$
\mathcal{R}\left(f\left(U^{-1} \cdot\right)\right)=U \mathcal{R} f\left(U^{-1} \cdot\right) .
$$

The corresponding $\mathcal{R}$-complex signal of $f \in L_{2}\left(\mathbb{R}^{n}, \mathbb{R}\right)$ is called monogenic signal, see [7].

In the following we are interested in the case $n=2$. Then the we can alternatively consider the $2 D$ complex Riesz operator $\mathcal{R}: L_{2}\left(\mathbb{R}^{2}, \mathbb{R}\right) \rightarrow L_{2}\left(\mathbb{R}^{2}, \mathbb{C}\right)$ defined by the convolution

$$
\mathcal{R} f(x):=\left(\frac{1}{2 \pi} \frac{x_{1}^{\prime}+i x_{2}^{\prime}}{\left|x^{\prime}\right|^{3}} * f\right)(x),
$$

respectively, in the Fourier domain by

$$
\widehat{\mathcal{R} f}(\omega):=\frac{-i \omega_{1}+\omega_{2}}{|\omega|} \hat{f}(\omega)=-i e^{i \varphi(\omega)} \hat{f}(\omega), \quad \omega:=\omega_{1}+i \omega_{2}
$$

where $\varphi \in(-\pi, \pi]$ is given by

$$
\varphi(\omega)=\operatorname{atan} 2\left(\omega_{1}, \omega_{2}\right):=\left\{\begin{aligned}
\arccos \frac{\omega_{1}}{|\omega|} & \text { if } \omega_{2} \geq 0, \\
-\arccos \frac{\omega_{1}}{|\omega|} & \text { if } \omega_{2}<0 .
\end{aligned}\right.
$$

By (5) we obtain for the rotation matrix $R_{\theta}:=\left(\begin{array}{rr}\cos \theta & -\sin \theta \\ \sin \theta & \cos \theta\end{array}\right)$ the rotation covariance relation of the Riesz transform

$$
\mathcal{R}\left(f\left(R_{\theta}^{-1} \cdot\right)\right)=e^{i \theta} \mathcal{R} f\left(R_{\theta}^{-1} \cdot\right) .
$$

The monogenic signal is defined by

$$
f_{\mathrm{m}}:=\mathcal{R}^{\prime} f=\left(f, f_{1}, f_{2}\right), \quad f_{1}:=\mathcal{R}_{1} f, \quad f_{2}:=\mathcal{R}_{2} f .
$$

In agreement with (3) it has the amplitude

$$
A:=\sqrt{f^{2}+f_{1}^{2}+f_{2}^{2}}
$$

The local orientation $\theta \in(-\pi, \pi]$ and instantaneous phase $\xi \in[0, \pi]$ are specified via

$$
f=A \cos \xi, \quad f_{1}=A \sin \xi \cos \theta, \quad f_{2}=A \sin \xi \sin \theta .
$$

In accordance with (4) the instantaneous phase is recovered by

$$
\xi=\arccos \frac{f}{A} .
$$

With $r:=\sqrt{f_{1}^{2}+f_{2}^{2}}=A \sin (\xi)$ we obtain the local orientation vector by $\left(\frac{f_{1}}{r}, \frac{f_{2}}{r}\right)=(\cos \theta, \sin \theta)^{\mathrm{T}}$ and the local orientation by

$$
\theta:=\operatorname{atan} 2\left(f_{1}, f_{2}\right)
$$




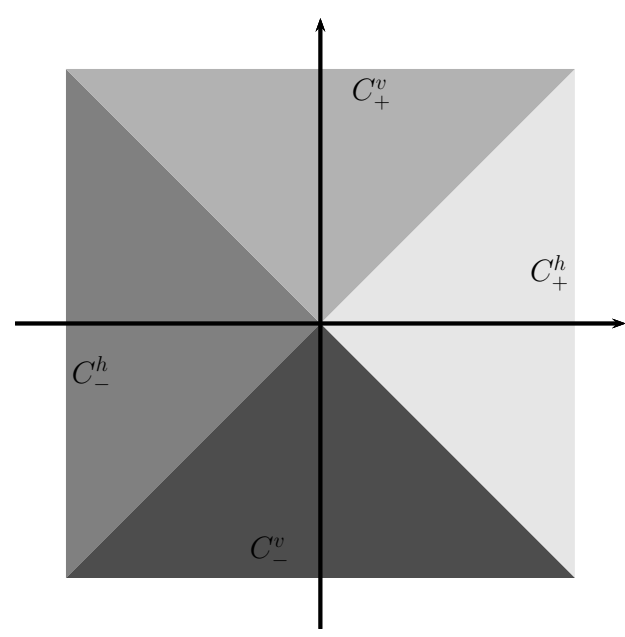

(a) The (monogenic) cones $C_{h}^{ \pm}$and $C_{v}^{ \pm}$

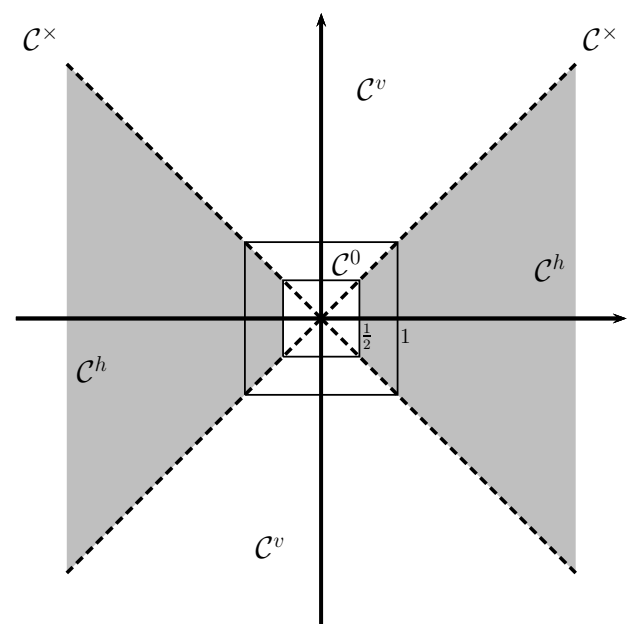

(b) The (shearlet) cones $\mathcal{C}^{h}, \mathcal{C}^{v}, \mathcal{C}^{\times}$and $\mathcal{C}^{0}$

Figure 2: The "monogenic cones" (left) and the "shearlet" cones (right).

\section{$3 \quad$ Linearized Riesz transform and quasi-monogenic signals}

In this section we are interested in a quadrature operator having a similar property (7) as the Riesz transform but with respect to the shear operator

$$
S_{s}:=\left(\begin{array}{cc}
1 & s \\
0 & 1
\end{array}\right), \quad s \in \mathbb{R}
$$

\subsection{Definition and Properties}

Let

$$
\begin{aligned}
& C_{+}^{v}:=\left\{\omega \in \mathbb{R}^{2}:\left|\frac{\omega_{2}}{\omega_{1}}\right| \leq 1, \omega_{1} \geq 0\right\}, C_{-}^{v}:=\left\{\omega \in \mathbb{R}^{2}:\left|\frac{\omega_{2}}{\omega_{1}}\right| \leq 1, \omega_{1} \leq 0\right\}, C^{v}:=C_{+}^{v} \cup C_{-}^{v}, \\
& C_{+}^{h}:=\left\{\omega \in \mathbb{R}^{2}:\left|\frac{\omega_{1}}{\omega_{2}}\right| \leq 1, \omega_{2} \geq 0\right\}, C_{-}^{h}:=\left\{\omega \in \mathbb{R}^{2}:\left|\frac{\omega_{1}}{\omega_{2}}\right| \leq 1, \omega_{2} \leq 0\right\}, C^{h}:=C_{+}^{h} \cup C_{-}^{h},
\end{aligned}
$$

see Fig. 2 left. We introduce the linearized Riesz transform $\mathcal{R}_{L}: L_{2}\left(\mathbb{R}^{2}, \mathbb{R}\right) \rightarrow L_{2}\left(\mathbb{R}^{2}, \mathbb{C}\right)$ by

$$
\widehat{\mathcal{R}_{L} f}(\omega):=-i e^{i \varphi_{L}(\omega)} \hat{f}(\omega),
$$

where

$$
\begin{aligned}
\varphi_{L}(\omega):= \begin{cases}\left(1-\operatorname{sgn}\left(\omega_{1}\right)\right) \operatorname{sgn}\left(\omega_{2}\right) \frac{\pi}{2}+\frac{\omega_{2}}{\omega_{1}} \frac{\pi}{4} & \text { if }\left(\omega_{1}, \omega_{2}\right) \in C^{h}, \\
\operatorname{sgn}\left(\omega_{2}\right) \frac{\pi}{2}-\frac{\omega_{1}}{\omega_{2}} \frac{\pi}{4} & \text { if }\left(\omega_{1}, \omega_{2}\right) \in C^{v},\end{cases} \\
= \begin{cases}\frac{\omega_{2}}{\omega_{1}} \frac{\pi}{4} & \text { if }\left(\omega_{1}, \omega_{2}\right) \in C_{+}^{h}, \\
\frac{\pi}{2}-\frac{\omega_{1}}{\omega_{2}} \frac{\pi}{4} & \text { if }\left(\omega_{1}, \omega_{2}\right) \in C_{+}^{v}, \\
\operatorname{sgn}\left(\omega_{2}\right) \pi+\frac{\omega_{2}}{\omega_{1}} \frac{\pi}{4} & \text { if }\left(\omega_{1}, \omega_{2}\right) \in C_{-}^{h}, \\
-\frac{\pi}{2}-\frac{\omega_{1}}{\omega_{2}} \frac{\pi}{4} & \text { if }\left(\omega_{1}, \omega_{2}\right) \in C_{-}^{v} .\end{cases}
\end{aligned}
$$




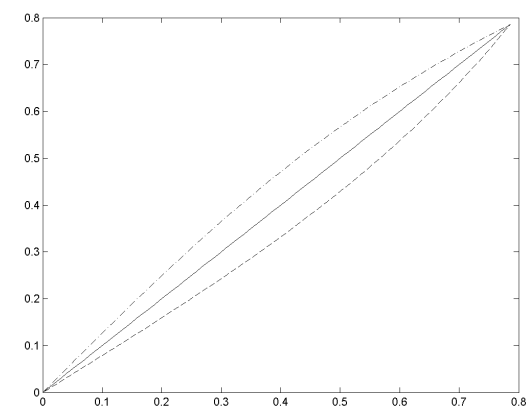

Figure 3: Function $\varphi(x)=x$ for $x \in\left[0, \frac{\pi}{4}\right]$ (solid line), our approximation $\varphi_{L}(x)=\frac{\pi}{4} \tan x$ (point-dotted line) and $\varphi_{\text {equi }}=\arctan \frac{4}{\pi} x$ (dotted line).

Note that $\varphi=\arctan y, y \in[0,1]$ implies $\varphi_{L}=\frac{\pi}{4} y=\frac{\pi}{4} \tan \varphi$. Fig. 3 shows the function $\varphi(x)=x$ for $x \in\left[0, \frac{\pi}{4}\right]$ versus our approximation $\varphi_{l}(x)=\frac{\pi}{4} \tan x$ and $\varphi_{\text {equi }}=\arctan \frac{4}{\pi} x$. Corresponding to (2) we define $\mathcal{R}_{L}: L_{2}\left(\mathbb{R}^{2}, \mathbb{R}\right) \rightarrow L_{2}\left(\mathbb{R}^{2}, \mathbb{R}^{2}\right)$ by

$$
\mathcal{R}_{L} f=\mathbf{e}_{1} \mathcal{R}_{L, 1} f+\mathbf{e}_{2} \mathcal{R}_{L, 2} f,
$$

where

$$
\widehat{\mathcal{R}_{L, 1} f}(\omega):=-i \cos \varphi_{L}(\omega) \hat{f}(\omega), \quad \widehat{\mathcal{R}_{L, 2} f}(\omega):=-i \sin \varphi_{L}(\omega) \hat{f}(\omega) .
$$

Fig. 4 shows the linearized phase $\varphi_{L}$, the original phase $\varphi$ and the respective differences to the original Riesz transform.

Proposition 3.1. The linearized Riesz transform is a quadrature operator of second order.

Proof. The proof follows directly from the definition of the linearized Riesz transform.

1. We see that $\mathcal{R}_{L}$ is translation invariant by

$$
\begin{aligned}
\mathcal{R}_{L}(f(\cdot-t))^{\wedge}(\omega) & =i e^{i \varphi_{L}(\omega)}(f(\cdot-t))^{\wedge}(\omega)=i e^{i \varphi_{L}(\omega)} e^{-2 \pi i t \omega} \hat{f}(\omega), \\
\left(\left(\mathcal{R}_{L} f\right)(\cdot-t)\right)^{\wedge}(\omega) & =e^{-2 \pi i t \omega} \widehat{\mathcal{R}_{L} f}(\omega)=e^{-2 \pi i t \omega} i e^{i \varphi_{L}(\omega)} \hat{f}(\omega) .
\end{aligned}
$$

2. Next, $\mathcal{R}_{L}$ is invariant with respect to dilations by $\alpha>0$ since

$$
\begin{aligned}
\mathcal{R}_{L}(f(\alpha \cdot))^{\wedge}(\omega) & =i e^{i \varphi_{L}(\omega)}(f(\alpha \cdot))^{\wedge}(\omega)=i e^{i \varphi_{L}(\omega)} \frac{1}{\alpha} \hat{f}\left(\frac{\omega}{\alpha}\right), \\
\left(\left(\mathcal{R}_{L} f\right)(\alpha \cdot)\right)^{\wedge}(\omega) & =\frac{1}{\alpha} \widehat{\mathcal{R}_{L} f}\left(\frac{\omega}{\alpha}\right)=\frac{1}{\alpha} i e^{i \varphi_{L}(\omega / \alpha)} \hat{f}\left(\frac{\omega}{\alpha}\right)
\end{aligned}
$$

and $\varphi_{L}(\omega / \alpha)=\varphi_{L}(\omega)$ for $\alpha>0$.

3 . We have by (1) that $\mathcal{R}_{L}$ is self-inverting since

$$
\mathcal{R}_{L}\left(\mathcal{R}_{L} f\right)=\mathcal{R}_{L}\left(\boldsymbol{e}_{1} \mathcal{R}_{L, 1} f+\boldsymbol{e}_{2} \mathcal{R}_{L, 2} f\right)=-\left(\mathcal{R}_{L, 1}^{2} f+\mathcal{R}_{L, 2}^{2} f\right)
$$

and therefore

$$
\left.\widehat{\mathcal{R}_{L}\left(\mathcal{R}_{L}\right.} f\right)(\omega)=-\left(-i \cos \varphi_{L}(\omega) \widehat{\mathcal{R}_{L, 1} f}(\omega)-i \sin \varphi_{L}(\omega) \widehat{\mathcal{R}_{L, 2} f}(\omega)\right)=\hat{f}(\omega) .
$$


4. Finally using (13) we see that $\mathcal{R}_{L}$ is anti-selfadjoint since for $\mathcal{R}_{L, 1}$ (and similarly for $\mathcal{R}_{L, 2}$ ),

$$
\left\langle f, \mathcal{R}_{L, 1} g\right\rangle=\left\langle\hat{f}, \widehat{\mathcal{R}_{L, 1} g}\right\rangle=\left\langle\hat{f},-i \cos \varphi_{L} \hat{g}\right\rangle=\left\langle i \cos \varphi_{L} \hat{f}, \hat{g}\right\rangle=\left\langle-\mathcal{R}_{L, 1} f, g\right\rangle .
$$

Now we define the the quasi-monogenic signal of $f \in L_{2}\left(\mathbb{R}^{2}, \mathbb{R}\right)$ to be

$$
f_{\mathrm{qm}}:=\left(f, f_{L, 1}, f_{L, 2}\right), \quad f_{L, 1}=\mathcal{R}_{L, 1} f, \quad f_{L, 2}=\mathcal{R}_{L, 2} f .
$$

The local amplitude, instantaneous phase and local orientation are defined as for the monogenic signal (see (8), (9) and (10) ) but with respect to $f_{L, 1}$ and $f_{L, 2}$ now. Alternatively we could define the local orientation adapted to the linearized Riesz transform as in (12). In this paper we focus on the first version.

Finally, are interested in the relation between $\mathcal{R}_{L}$ and the shear operator. With respect to our shearlet filters in the next section we only consider functions $g$ supported on a subset of the cones $C^{\kappa}, \kappa \in\{h, v\}$.

Proposition 3.2. Let $g \in L_{2}\left(\mathbb{R}^{2}, \mathbb{R}\right)$ be a filter function such that $\hat{g}$ is supported in $\left\{\omega \in \mathbb{R}^{2}\right.$ : $\left.\left|\frac{\omega_{2}}{\omega_{1}}\right| \leq \alpha\right\}$, where $0 \leq \alpha<1$. Then, for $\alpha-1 \leq s \leq 1-\alpha$, the relation

$$
\mathcal{R}_{L}\left(g\left(S_{s}^{-1} \cdot\right)\right)=e^{i s \frac{\pi}{4}}\left(\mathcal{R}_{L} g\right)\left(S_{s}^{-1} \cdot\right)
$$

holds true. In other words, up to a rotation by the shear factor $s \frac{\pi}{4}$, the linearized Riesz transform of the sheared filter equals the sheared linearized Riesz transform of the filter.

Note that the restriction on the shear parameter is in agreement with our later shearlet construction by (cone restricted) shearing of a mother shearlet.

Proof. By definition of $\mathcal{R}_{L}$ we have

$$
\begin{aligned}
\left(\mathcal{R}_{L} g\left(S_{s}^{-1} \cdot\right)\right)^{\wedge}(\omega) & =-i e^{i \varphi_{L}(\omega)} \hat{g}\left(S_{s}^{\mathrm{T}} \omega\right) \\
& =-i e^{i \varphi_{L}(\omega)} \hat{g}\left(\omega_{1}, s \omega_{1}+\omega_{2}\right) .
\end{aligned}
$$

By the support assumption on $g$, the above function becomes zero if the following condition is not fulfilled:

$$
-\alpha \leq s+\frac{\omega_{2}}{\omega_{1}} \leq \alpha
$$

By assumption on $s$ this implies $-1 \leq-\alpha-s \leq \frac{\omega_{2}}{\omega_{1}} \leq \alpha-s \leq 1$ and we get for $\omega_{1} \geq 0$ by definition of $\varphi_{L}$ the relation

$$
\left(\mathcal{R}_{L} g\left(S_{s}^{-1} \cdot\right)\right)^{\wedge}(\omega)=-i e^{i \frac{\omega_{2}}{\omega_{1}} \frac{\pi}{4}} \hat{g}\left(S_{s}^{\mathrm{T}} \omega\right) .
$$

On the other hand, we obtain

$$
\begin{aligned}
\left(\left(\mathcal{R}_{L} g\right)\left(S^{-1} \cdot\right)\right)^{\wedge}(\omega) & =\left(\mathcal{R}_{L} g\right)^{\wedge}\left(S_{s}^{\mathrm{T}} \omega\right) \\
& =-i e^{i \varphi_{L}\left(S_{s}^{\mathrm{T}} \omega\right)} \hat{g}\left(S_{s}^{\mathrm{T}} \omega\right),
\end{aligned}
$$


where in case $\omega_{1} \geq 0$,

$$
e^{i \varphi_{L}\left(S_{s}^{\mathrm{T}} \omega\right)}=e^{i\left(s+\frac{\omega_{2}}{\omega_{1}}\right) \frac{\pi}{4}}=e^{i s \frac{\pi}{4}} e^{i \varphi_{L}(\omega)}
$$

so that

$$
\left(\left(\mathcal{R}_{L} g\right)\left(S^{-1} \cdot\right)\right)^{\wedge}(\omega)=e^{i s \frac{\pi}{4}}\left(\mathcal{R}_{L} g\left(S_{s}^{-1} \cdot\right)\right)^{\wedge}(\omega) .
$$

Similarly we can conclude for $\omega_{1}<0$. Then the assertion follows by taking the inverse Fourier transform.

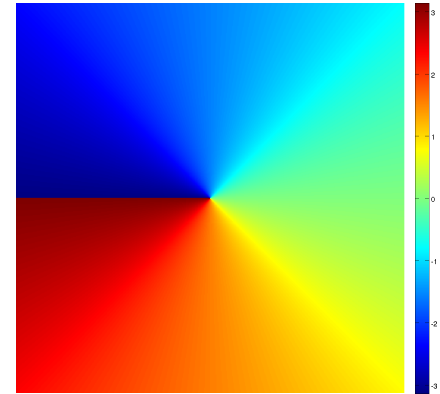

(a) $\varphi_{L}$

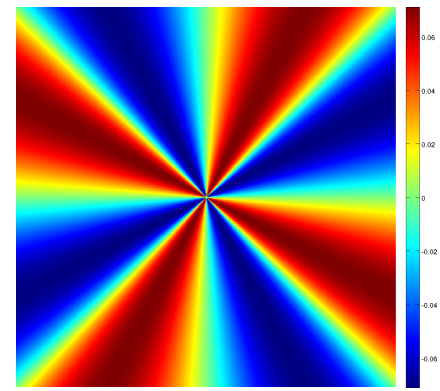

(c) $\varphi-\varphi_{L}$

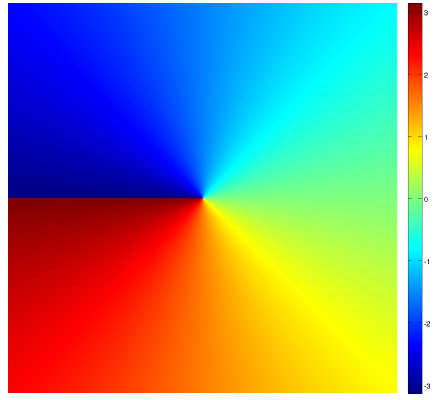

(b) $\varphi$

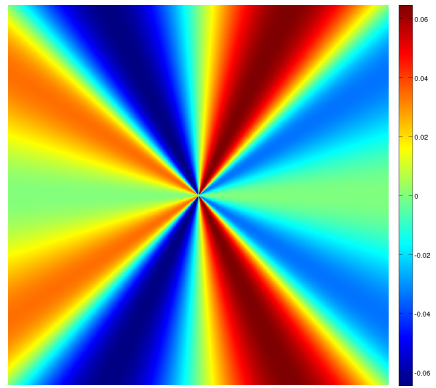

(d) $\Re\left(e^{i \varphi}-e^{i \varphi_{L}}\right)$

Figure 4: Comparison of linearized and usual Riesz operators.

\subsection{Linearized Riesz transform versus Riesz transform}

In this subsection we compare the linearized Riesz transform and the ordinary Riesz transform by a numerical example and by an optical filtering devise.

A Numerical Comparison. First we deal with the synthetic image in Fig. 5. The Riesz components $\mathcal{R}_{L, 1} f$ and $\mathcal{R}_{L, 2} f$ are depicted in Fig. 6 together with the difference to the original Riesz transformed image.

The amplitude, instantaneous phase and local orientation are shown in Figure 7 . Since the latter two are only well defined at points with a significant amplitude we threshold them and show only entries where the amplitude is larger that $\mathrm{T} \%$ of the maximal amplitude and the threshold $\mathrm{T}$ is given with the images. 


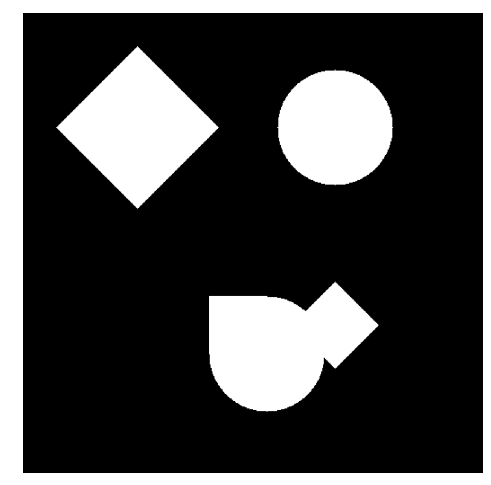

Figure 5: Test image $f$.

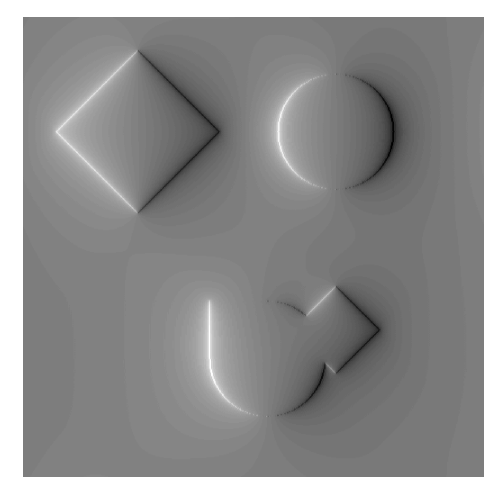

(a) $\mathcal{R}_{L, 1} f$

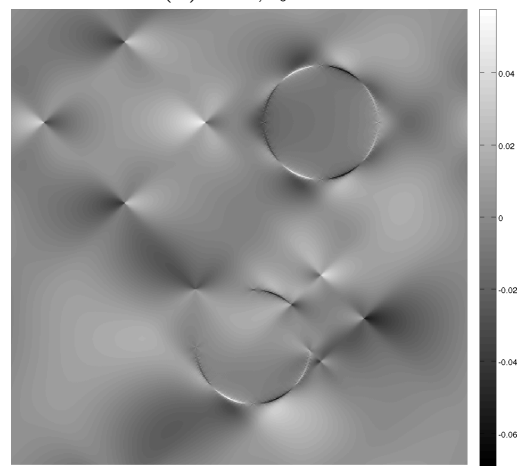

(c) $\mathcal{R}_{1} f-\mathcal{R}_{L, 1} f$

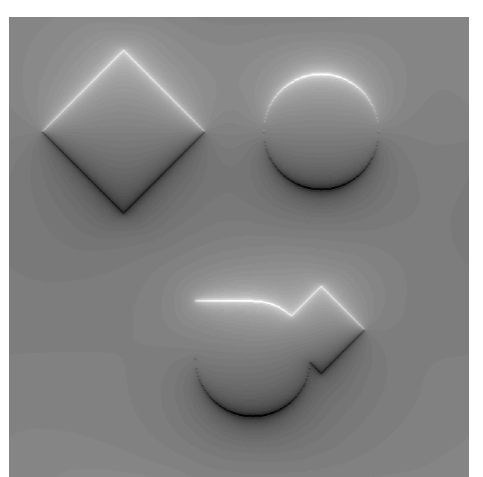

(b) $\mathcal{R}_{L, 2} f$

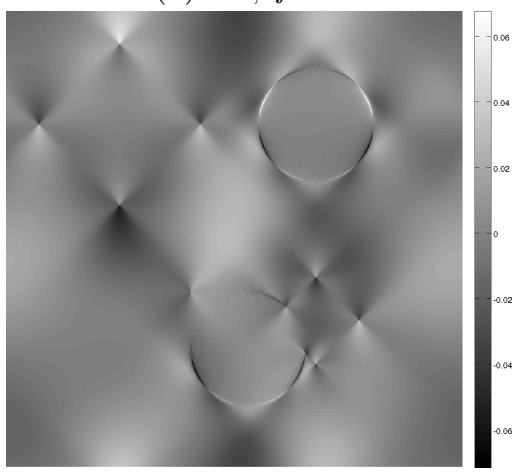

(d) $\mathcal{R}_{2} f-\mathcal{R}_{L, 2} f$

Figure 6: Comparison of linearized and ordinary Riesz transform. 


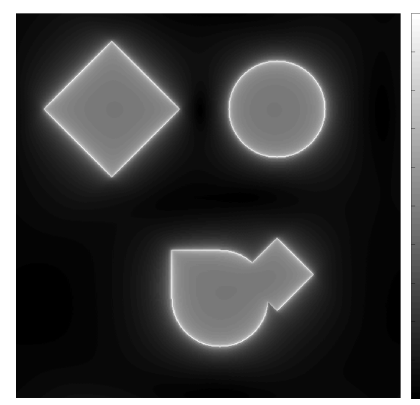

(a) Amplitude $A_{\text {lin }}$

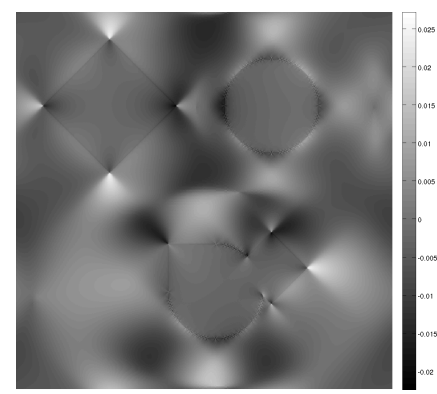

(d) Difference Amplitude $A-$ $A_{\text {lin }}$

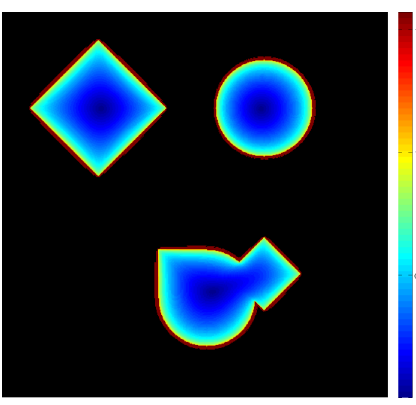

(b) Phase $\xi_{\text {lin }}(T=40)$

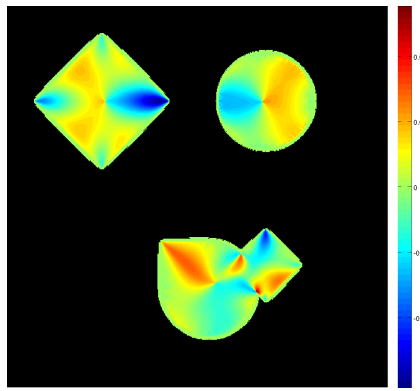

(e) Difference Phase $\xi-\xi_{\text {lin }}$

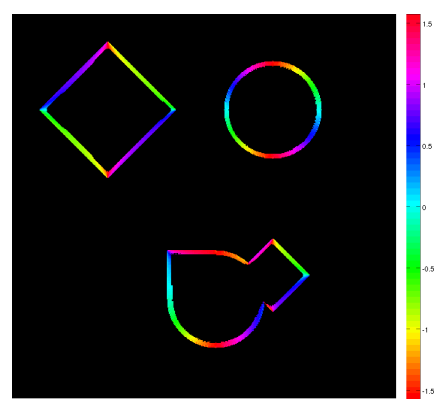

(c) Orientation $\theta_{\operatorname{lin}}(T=60)$

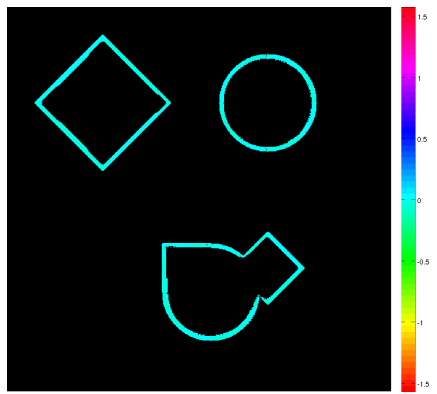

(f) Difference Orientation $\theta-$ $\theta_{\text {lin }}$

Figure 7: Local amplitude, instantaneous phase and orientation of the test image obtained by the linearized Riesz transform.

An optical filter comparison. In optics, spiral phase (SP) filtering [1, 24] provides an experimental analogy to the application of the (2D) Riesz transform kernel in mathematics. An optical implementation can be performed by introducing a filter, here realized by a spatial light modulator (SLM), in the focal plane of a Fourier plane filtering (FPF) unit [10]. Addressing the SLM by the Fourier filter function:

$$
H(\omega)=e^{i \varphi(\omega)}
$$

with the usual azimuthal angle $\varphi(\omega)$, an optically filtered image of modified contrast can be recorded by a camera system [23].

The expression in (14), characterizing a conventional SP filter, corresponds in its formal structure to the Fourier multiplier of the 2D Riesz transform $\widehat{\mathcal{R f}}(\omega)$ in $(6)$. In analogy, a (so-called) linearized SP filter is characterized by the Fourier filter function

$$
H_{L}(\omega)=e^{i \varphi_{L}(\omega)}
$$

with the linearized azimuthal angle $\varphi_{L}(\omega)$ defined in (12). The expression in (15) corresponds to the Fourier multiplier of the 2D linearized Riesz transform $\widehat{\mathcal{R}_{L} f}(\omega)$ in (11).

The linearized SP filter can be addressed on the SLM as well. Both types of realized SP filters deliver an almost isotropic contrast modification and a possibility for an optical edge detection of the investigated micro-structures, in an analog manner as described for edge detection by analytic signal approaches in image processing [14, 2].

In Fig. 8(a)-(e) the measured (intensity) data after applying the conventional and linearized SP filter are depicted, with only minor differences between their filtering results. The applied 


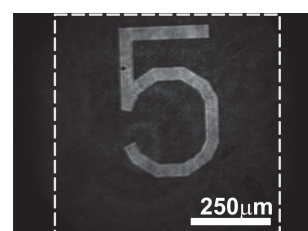

(a) test structure without filtering

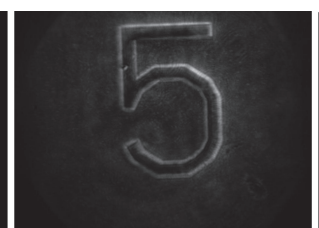

(b) ordinary SP filtered

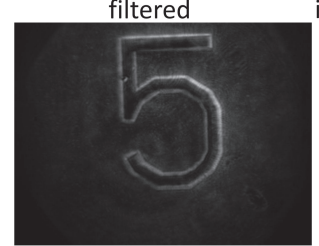

(c) linearized SP filtered

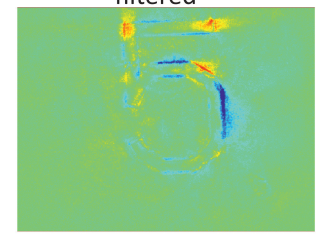

(d) difference ((b)-(c))

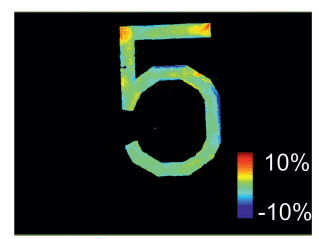

(e) overlay of (d) with binarized mask (a)

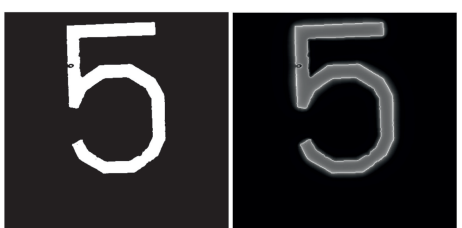

(f) binarized test (g) local amplitude of image (dashed (a)) ordinary RT

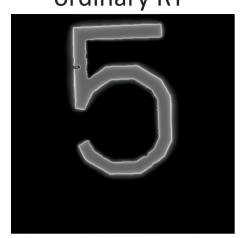

(h) local amplitude of linearized RT

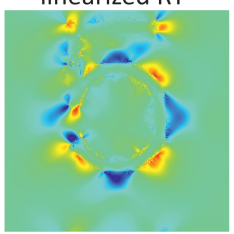

(i) difference ((g)-(h))

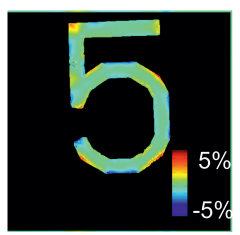

(j) overlay of (i) with binarized mask (f)

Figure 8: Analogy between optical and mathematical approaches for isotropic edge enhancement/detection:

Conventional and linearized spiral phase (SP) filtering, applied as an optical method on a test structure (metalized 'Number 5'), ((a)-(e)).

Ordinary and linearized Riesz transform (RT) approach, applied as a mathematical method on a test image (binarized 'Number 5'), ((f)-(j)).

SLM has been a liquid crystal phase-only type (SLM Pluto, HoloEye AG). Additionally, the local amplitude computed by the ordinary and linearized Riesz transform approach are shown for a binarized test image in Fig. 8(f)-(j).

\section{Shearlets on the cone and their quasi-monogenic version}

Let $\psi \in L_{2}\left(\mathbb{R}^{2}, \mathbb{R}\right)$ be a function fulfilling

$$
\hat{\psi}(\omega)=\hat{\psi}_{1}\left(\omega_{1}\right) \hat{\psi}_{2}\left(\frac{\omega_{2}}{\omega_{1}}\right),
$$


where $\psi_{1}$ is a wavelet and $\hat{\psi}_{2}$ a continuous bump function with $\operatorname{supp} \psi_{2} \subseteq[-1,1]$. Typical choices for $\psi_{1}$ and $\psi_{2}$ are

$$
\hat{\psi}_{1}\left(\omega_{1}\right):=\sqrt{b^{2}\left(2 \omega_{1}\right)+b^{2}\left(\omega_{1}\right)} \quad \text { and } \quad \hat{\psi}_{2}\left(\omega_{2}\right):= \begin{cases}\sqrt{v\left(1+\omega_{2}\right)} & \text { for } \omega_{2} \leq 0 \\ \sqrt{v\left(1-\omega_{2}\right)} & \text { for } \omega_{2}>0\end{cases}
$$

where

$$
v(x):= \begin{cases}0 & \text { for } x<0 \\ 35 x^{4}-84 x^{5}+70 x^{6}-20 x^{7} & \text { for } 0 \leq x \leq 1, \\ 1 & \text { for } x>1\end{cases}
$$

and

$$
b(x):= \begin{cases}\sin \left(\frac{\pi}{2} v(|x|-1)\right) & \text { for } 1 \leq|x| \leq 2 \\ \cos \left(\frac{\pi}{2} v\left(\frac{1}{2}|x|-1\right)\right) & \text { for } 2<|x| \leq 4 \\ 0 & \text { otherwise }\end{cases}
$$

see $[22,21]$. Such a function fulfills the admissibility condition

$$
\int_{\mathbb{R}^{2}} \frac{\left|\hat{\psi}\left(\omega_{1}, \omega_{2}\right)\right|^{2}}{\omega_{1}^{2}} d \omega_{1} d \omega_{2}<\infty
$$

and will serve as mother shearlet. Let the parabolic scaling matrix $A_{a}$ be defined by

$$
A_{a}=\left(\begin{array}{cc}
a & 0 \\
0 & \sqrt{a}
\end{array}\right), \quad a \in \mathbb{R}^{+} .
$$

Then the shearlets $\psi_{a, s, t}$ emerge by dilation, shearing and translation

$$
\psi_{a, s, t}(x):=a^{-\frac{3}{4}} \psi\left(A_{a}^{-1} S_{s}^{-1}(x-t)\right),
$$

see $[11,19]$. In the Fourier domain this reads

$$
\hat{\psi}_{a, s, t}(\omega)=a^{\frac{3}{4}} e^{-2 \pi i\langle t, \omega\rangle} \hat{\psi}\left(A_{a}^{\mathrm{T}} S_{s}^{\mathrm{T}} \omega\right)=a^{\frac{3}{4}} e^{-2 \pi i\langle t, \omega\rangle} \hat{\psi}\left(a \omega_{1}, \sqrt{a}\left(s \omega_{1}+\omega_{2}\right)\right) .
$$

The continuous shearlet transform $\mathcal{S H}_{\psi}(f)$ of a function $f \in L_{2}(\mathbb{R})$ is defined by

$$
\mathcal{S H}_{\psi}(f)(a, s, t):=\left\langle f, \psi_{a, s, t}\right\rangle=\left\langle\hat{f}, \hat{\psi}_{a s t}\right\rangle .
$$

For practical computations we are interested in shearlets on the cone. To this end we define the restricted horizontal and the vertical cones by

$$
\begin{aligned}
& \mathcal{C}^{h}:=\left\{\left(\omega_{1}, \omega_{2}\right) \in \mathbb{R}^{2}:\left|\omega_{1}\right| \geq \frac{1}{2},\left|\omega_{2}\right|<\left|\omega_{1}\right|\right\}, \\
& \mathcal{C}^{v}:=\left\{\left(\omega_{1}, \omega_{2}\right) \in \mathbb{R}^{2}:\left|\omega_{2}\right| \geq \frac{1}{2},\left|\omega_{2}\right|>\left|\omega_{1}\right|\right\},
\end{aligned}
$$

resp., and the "intersection" (seam lines) of the two cones and the "low frequency" set by

$$
\begin{aligned}
\mathcal{C}^{\times} & :=\left\{\left(\omega_{1}, \omega_{2}\right) \in \mathbb{R}^{2}:\left|\omega_{1}\right| \geq \frac{1}{2},\left|\omega_{2}\right| \geq \frac{1}{2},\left|\omega_{1}\right|=\left|\omega_{2}\right|\right\}, \\
\mathcal{C}^{0} & :=\left\{\left(\omega_{1}, \omega_{2}\right) \in \mathbb{R}^{2}:\left|\omega_{1}\right|<1,\left|\omega_{2}\right|<1\right\},
\end{aligned}
$$


resp., see Fig. 2, right. Altogether $\mathbb{R}^{2}=\mathcal{C}^{h} \cup \mathcal{C}^{v} \cup \mathcal{C}^{\times} \cup \mathcal{C}^{0}$ with an overlapping domain $C^{\square}:=(-1,1)^{2} \backslash\left(-\frac{1}{2}, \frac{1}{2}\right)^{2}$. To obtain a discrete shearlets on the cone, we discretize the scaling and shear parameters as

$$
\begin{aligned}
a_{j} & :=2^{-2 j}=\frac{1}{4^{j}}, \quad j=0, \ldots, j_{0}-1, \\
s_{j, k} & :=k 2^{-j}, \quad-2^{j} \leq k \leq 2^{j}, k \in \mathbb{Z},
\end{aligned}
$$

and specify the translation parameters $t_{m}, m \in \mathbb{Z}$ later. With these notations our shearlets $\psi^{h}$ on the horizontal cone $\mathcal{C}^{h}$ are given in the frequency domain by

$$
\hat{\psi}_{j, k, m}^{h}(\omega)=2^{3 j / 2} \hat{\psi}^{h}\left(A_{a_{j}}^{\mathrm{T}} S_{s_{j, k}}^{\mathrm{T}} \omega\right) e^{-2 \pi i\langle\omega, m\rangle}=\hat{\psi}_{1}\left(4^{-j} \omega_{1}\right) \hat{\psi}_{2}\left(2^{j} \frac{\omega_{2}}{\omega_{1}}+k\right) e^{-2 \pi i\left\langle\omega, t_{m}\right\rangle}
$$

and in the time domain by

$$
\psi_{j, k, m}^{h}(x):=2^{-3 j / 2} \psi_{a_{j}, s_{j k}, t_{m}}^{h}(x)=\psi^{h}\left(A_{a_{j}}^{-1} S_{s_{j k}}^{-1}\left(x-t_{m}\right)\right) .
$$

For $k=-2^{j}+1, \ldots, 2^{j}-1$ the shearlets $\hat{\psi}^{h}$ are supported on $\mathcal{C}^{h}$. In particular we see that $\hat{\psi}_{j, 0,0}^{h}$ is supported in $\left\{\omega \in \mathbb{R}^{2}:\left|\frac{\omega_{2}}{\omega_{1}}\right| \leq 2^{-j}\right\}$. Hence, using $\alpha=2^{-j}$ and $s=2^{-j} k$ in Proposition 3.2 we obtain

$$
\mathcal{R}_{L}\left(\psi_{j, 0,0}\left(S_{s_{j k}}^{-1} \cdot\right)\right)=e^{2^{-j} k \frac{\pi}{4}}\left(\mathcal{R}_{L} \psi_{j, 0,0}\right)\left(S_{s_{j k}}^{-1} \cdot\right) .
$$

The shearlets $\psi^{v}$ on the vertical cone are defined by changing the roles of $\omega_{1}$ and $\omega_{2}$ in (16). Moreover, we define

$$
\psi_{j, \pm 2^{j}, m}^{h \times v}:=\psi_{j, \pm 2^{j}, m}^{h} \chi_{\mathcal{C}^{\times}}
$$

with the characteristic function $\chi_{C}$ which is equal to 1 for $\omega \in C$ and 0 otherwise. (Note that the separate consideration of $\mathcal{C}^{\times}$is only useful in the later discrete setting.) Finally, we use the scaling function $\phi$ defined by

$$
\hat{\phi}\left(\omega_{1}, \omega_{2}\right):= \begin{cases}\varphi\left(\omega_{1}\right) & \text { for }\left|\omega_{1}\right|<1,\left|\omega_{2}\right| \leq\left|\omega_{1}\right| \\ \varphi\left(\omega_{2}\right) & \text { for }\left|\omega_{2}\right|<1,\left|\omega_{1}\right|<\left|\omega_{2}\right|\end{cases}
$$

with

$$
\varphi(\omega):= \begin{cases}1 & \text { for }|\omega| \leq \frac{1}{2} \\ \cos \left(\frac{\pi}{2} v(2|\omega|-1)\right) & \text { for } \frac{1}{2}<|\omega|<1 \\ 0 & \text { otherwise }\end{cases}
$$

and its translates $\phi_{m}(x)=\phi\left(x-t_{m}\right)$, i.e., $\hat{\phi}_{m}(\omega)=e^{-2 \pi i\left\langle t_{m}, \omega\right\rangle} \hat{\phi}(\omega)$ on $\mathcal{C}^{0}$.

In image processing we work in a finite discrete setting. We consider digital images as functions sampled on the grid

$$
\frac{1}{N} \mathcal{I}:=\frac{1}{N}\left\{\left(m_{1}, m_{2}\right): m_{i}=0, \ldots, N-1, i=1,2\right\}
$$

and assume periodic continuation over the boundary. Let

$$
\Omega:=\left\{\left(\omega_{1}, \omega_{2}\right) \in \mathbb{Z}^{2}: \omega_{i}=-\left\lfloor\frac{N}{2}\right\rfloor, \ldots,\left\lceil\frac{N}{2}\right\rceil-1, i=1,2 .\right\} .
$$


Let $j_{0}:=\left\lfloor\frac{1}{2} \log _{2} N\right\rfloor$ be the number of considered scales, i.e., we deal only with the scales $j=0, \ldots, j_{0}$, and choose the translation parameters

$$
t_{m}:=\frac{m}{N}, \quad m \in \mathcal{I}
$$

Instead of the continuous Fourier transform we use the discrete Fourier transform (DFT) defined for $f: \mathcal{I} \rightarrow \mathbb{R}$ by

$$
\hat{f}(\omega)=\sum_{m \in \mathcal{I}} f(m) e^{-2 \pi i\langle\omega, m\rangle / N}, \quad \omega \in \Omega,
$$

and the inverse discrete Fourier transform (IDFT)

$$
f(m)=\frac{1}{N^{2}} \sum_{\omega \in \Omega} \hat{f}(\omega) e^{2 \pi i\langle\omega, m\rangle / N}, \quad m \in \mathcal{I} .
$$

We have the Plancherel formula with respect to the DFT

$$
\langle f, g\rangle=\frac{1}{N^{2}}\langle\hat{f}, \hat{g}\rangle .
$$

Recall that for a Hilbert space $\mathcal{H}$ a sequence $\left\{u_{j}: j \in \mathcal{J}\right\}$ is a tight frame if and only if there exists $A>0$ such that

$$
A\|f\|^{2}=\sum_{j \in \mathcal{J}}\left|\left\langle f, u_{j}\right\rangle\right|^{2} \quad \text { for all } f \in \mathcal{H} .
$$

For tight frames we have the reconstruction formula

$$
f=\frac{1}{A} \sum_{j \in \mathcal{J}}\left\langle f, u_{j}\right\rangle u_{j} \quad \text { for all } f \in \mathcal{H} .
$$

In [13], we have shown that the set

$$
\left\{\psi_{j, k, m}^{h}, \psi_{j, k, m}^{v}, \psi_{j, \pm 2^{j}, m}^{h \times v}: j=0, \ldots, j_{0}-1,-2^{j}+1 \leq k \leq 2^{j}-1, m \in \mathcal{I}\right\} \cup\left\{\phi_{m}: m \in \mathcal{I}\right\}
$$

provides a tight frame for $L_{2}(\mathcal{I})$ with $A=1$.

Let the linearized Riesz transform of a function $f: \mathcal{I} \rightarrow \mathbb{R}$ be defined via its DFT

$$
\widehat{\mathcal{R}_{L} f}(\omega):=-i e^{i \varphi_{L}(\omega)} \hat{f}(\omega), \quad \omega \in \Omega
$$

and let accordingly $\mathcal{R}_{L}^{\prime} f:=\left(f, \mathcal{R}_{L, 1} f, \mathcal{R}_{L, 2} f\right)$.

Then we define the discrete quasi-monogenic shearlets on the cone by

$$
\begin{aligned}
\mathcal{B}:= & \left\{\mathcal{R}_{L}^{\prime} \psi_{j, k, m}^{h}, \mathcal{R}_{L}^{\prime} \psi_{j, k, m}^{v}, \mathcal{R}_{L}^{\prime} \psi_{j, \pm 2^{j}, m}^{h \times v}: j=0, \ldots, j_{0}-1,-2^{j}+1 \leq k \leq 2^{j}-1, m \in \mathcal{I}\right\} \\
& \cup\left\{\mathcal{R}_{L}^{\prime} \phi_{m}: m \in \mathcal{I}\right\} .
\end{aligned}
$$

An example of quasi-monogenic shearlets is shown in Fig. 9.

Theorem 4.1. The set $\mathcal{B}$ of quasi-monogenic shearlets defined in (18) forms a tight frame for $L_{2}(\mathcal{I})$ with frame bound $A=2$. 


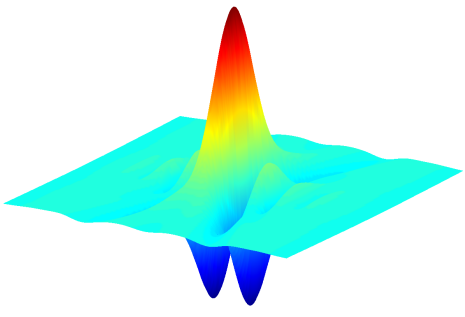

(a) 3D-view of $\psi_{1,0,0}^{h}$

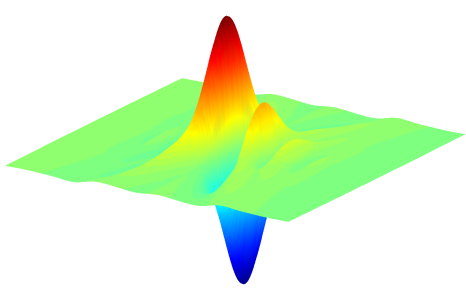

(b) 3 D-view of $\mathcal{R}_{1, L} \psi_{1,0,0}^{h}$

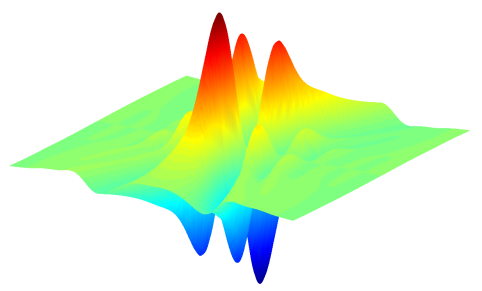

(c) 3D-view of $\mathcal{R}_{2, L} \psi_{1,0,0}^{h}$

Figure 9: Monogenic shearlet at scale $j=1\left(a=\frac{1}{4}\right)$.

Proof. We have to show that

$2\|f\|^{2}=\sum_{\kappa \in\{h, v\}} \sum_{j=0}^{j_{0}-1} \sum_{k=-2^{j}+1}^{2^{j}-1} \sum_{m \in \mathcal{I}}\left|\left\langle f, \mathcal{R}_{L}^{\prime} \psi_{j, k, m}^{\kappa}\right\rangle\right|^{2}+\sum_{j=0}^{j_{0}-1} \sum_{k= \pm 2^{j}} \sum_{m \in \mathcal{I}}\left|\left\langle f, \mathcal{R}_{L}^{\prime} \psi_{j, k, m}^{h \times v}\right\rangle\right|^{2}+\sum_{m \in \mathcal{I}}\left|\left\langle f, \phi_{m}\right\rangle\right|^{2}$.

By Parseval's identity (17) and definition (18) we obtain

$$
\begin{aligned}
\left|\left\langle f, \mathcal{R}_{L}^{\prime} \psi_{j, k, m}^{h}\right\rangle\right|^{2} & =\frac{1}{N^{2}}\left|\left\langle\hat{f}, \widehat{\mathcal{R}_{L}^{\prime} \psi_{j, k, m}^{h}}\right\rangle\right|^{2} \\
& =\left.\underbrace{\frac{1}{N} \sum_{\omega \in \Omega} \hat{f}(\omega) \hat{\psi}_{j, k, 0}^{h}(\omega) e^{2 \pi i\langle\omega, m\rangle / N}}_{=: g_{j, k}(m)}\right|^{2}+\left.\underbrace{\mid \frac{1}{N} \sum_{\omega \in \Omega} \hat{f}(\omega) \widehat{\mathcal{R}_{L} \psi_{j, k, 0}^{h}}(\omega) e^{2 \pi i\langle\omega, m\rangle / N}}_{=: \tilde{g}_{j, k}(m)}\right|^{2}
\end{aligned}
$$

and using Parseval's identity again

$$
\begin{aligned}
\sum_{m \in \mathcal{I}}\left|\left\langle f, \mathcal{R}_{L}^{\prime} \psi_{j, k, m}^{h}\right\rangle\right|^{2} & =\sum_{m \in \mathcal{I}}\left(\left|g_{j, k}(m)\right|^{2}+\left|\tilde{g}_{j, k}(m)\right|^{2}\right) \\
& =\frac{1}{N^{2}}\left(\sum_{\omega \in \Omega}\left|\hat{g}_{j, k}(\omega)\right|^{2}+\sum_{\omega \in \Omega}\left|\hat{\tilde{g}}_{j, k}(\omega)\right|^{2}\right) \\
& =\frac{1}{N^{2}}\left(\sum_{\omega \in \Omega}\left|\hat{g}_{j, k}(\omega)\right|^{2}+\sum_{\omega \in \Omega}\left|-i e^{i \varphi_{L}(\omega)} \hat{g}_{j, k}(\omega)\right|^{2}\right) \\
& =\frac{2}{N^{2}}\left(\sum_{\omega \in \Omega}|\hat{f}(\omega)|^{2}\left|\hat{\psi}_{j, k, 0}^{h}(\omega)\right|^{2}\right) \\
& =\frac{2}{N^{2}}\left(\sum_{\omega \in \Omega}\left|\hat{f}\left(\omega_{1}, \omega_{2}\right)\right|^{2}\left|\hat{\psi}_{1}\left(4^{-j} \omega_{1}\right)\right|^{2}\left|\hat{\psi}_{2}\left(k+2^{-j} \frac{\omega_{2}}{\omega_{1}}\right)\right|^{2}\right)
\end{aligned}
$$

The rest of the proof follows exactly the lines in [13].

Analogously to the discrete shearlet transform in [13] we define the quasi-monogenic discrete 
shearlet transform

$$
\mathcal{M S H}(f)(\kappa, j, k, m):= \begin{cases}\left\langle f, \phi_{m}\right\rangle & \text { for } \kappa=0, \\ \left\langle f, \mathcal{R}_{L}^{\prime} \psi_{j, k, m}^{\kappa}\right\rangle & \text { for } \kappa \in\{h, v\}, \\ \left\langle f, \mathcal{R}_{L}^{\prime} \psi_{j, k, m}^{h \times v}\right\rangle & \text { for } \kappa=\times,|k|=2^{j}\end{cases}
$$

where $j=0, \ldots, j_{0}-1,-2^{j}+1 \leq k \leq 2^{j}-1$ and $m \in \mathcal{I}$ if not stated in another way. For the computation of the quasi-monogenic shearlet transform we can use

$$
\begin{aligned}
\left\langle f, \mathcal{R}_{L}^{\prime} \psi_{j, k, m}^{\kappa}\right\rangle & =\left(\left\langle f, \psi_{j, k, m}^{\kappa}\right\rangle,\left\langle f, \mathcal{R}_{L, 1} \psi_{j, k, m}^{\kappa}\right\rangle,\left\langle f, \mathcal{R}_{L, 2} \psi_{j, k, m}^{\kappa}\right\rangle\right) \\
& =\left(\left\langle f, \psi_{j, k, m}^{\kappa}\right\rangle,\left\langle\mathcal{R}_{L, 1}^{*} f, \psi_{j, k, m}^{\kappa}\right\rangle,\left\langle\mathcal{R}_{L, 2}^{*} f, \psi_{j, k, m}^{\kappa}\right\rangle\right)
\end{aligned}
$$

which implies with $\left\langle f, \psi_{j, k,}^{\kappa},\right\rangle:=\left(\left\langle f, \psi_{j, k, m}^{\kappa}\right\rangle\right)_{m \in \mathcal{I}}$ that

$$
\left\langle f, \mathcal{R}_{L}^{\prime} \psi_{j, k, .}^{\kappa}\right\rangle=\left(\left\langle f, \psi_{j, k,}^{\kappa},\right\rangle, \mathcal{R}_{L, 1}^{*}\left\langle f, \psi_{j, k,}^{\kappa}\right\rangle, \mathcal{R}_{L, 2}^{*}\left\langle f, \psi_{j, k, \cdot}^{\kappa}\right\rangle\right),
$$

This means that we can just apply the adjoint Riesz transform to the shearlet coefficient images to obtain the monogenic coefficients.

In Fig. 10 we show the quasi-monogenic coefficients of the test image in Fig. 5 for selected scale and shear parameters. The small image at the lower left corner shows the support of the corresponding shearlet in the Fourier domain.

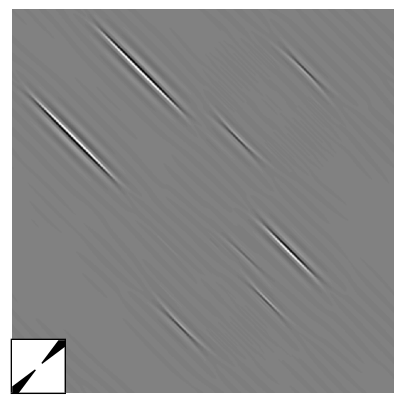

(a) $\left\langle f, \psi_{2,-4, .}^{h \times v}\right\rangle$

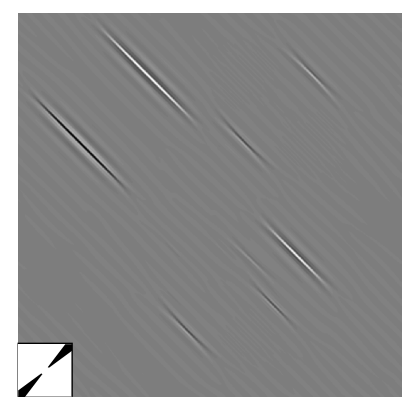

(b) $\mathcal{R}_{L, 1}^{*}\left\langle f, \psi_{2,-4, .}^{h \times v}\right\rangle$

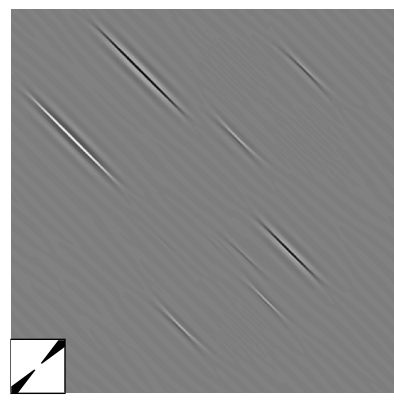

(c) $\mathcal{R}_{L, 2}^{*}\left\langle f, \psi_{2,-4,}^{h \times v}\right\rangle$

Figure 10: Quasi-monogenic shearlet coefficients at scale $j=2\left(a=\frac{1}{16}\right)$ and $k=-4(s=-1)$.

As for the Riesz transform we can compute the local amplitude, local orientation and instantaneous phase of the coefficients. This is shown in Fig. 11. The orientation and phase are thresholded with a fixed ratio of $80 \%$ of the maximal amplitude within each scale. Fig. 11(b) shows clearly the phase jump at the edges (see rhombus in the top left corner of the image). The orientation of the edges can be directly red from the colorbar (see Fig. 11(c)).

We conclude this section by showing the local orientation for several shearlets in Fig. 12.

\section{Numerical Examples of Texture Decomposition}

In this section we use the quasi-monogenic shearlet transform to analyse several texture images with inherent directional information.

We start with an image of a metal plate shown in Fig. 13(a). By considering amplitude, phase and orientation for different scale and shear parameters we can decompose the texture into its 


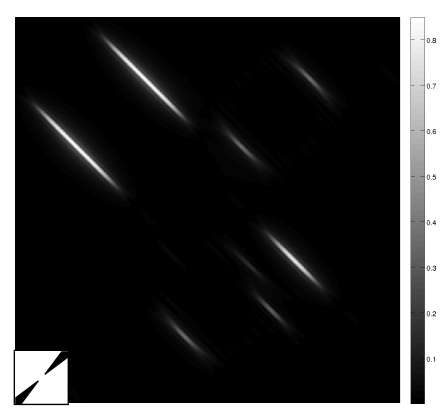

(a) Amplitude of the quasimonogenic shearlet coefficients

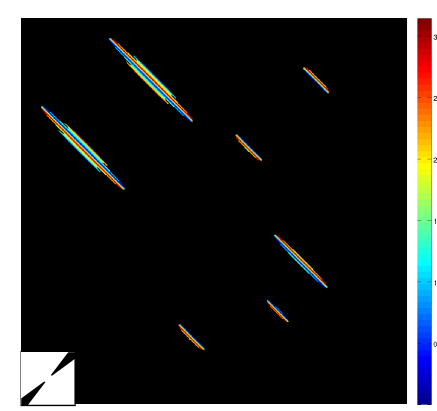

(b) Phase of the quasi-monogenic shearlet coefficients $(T=80)$

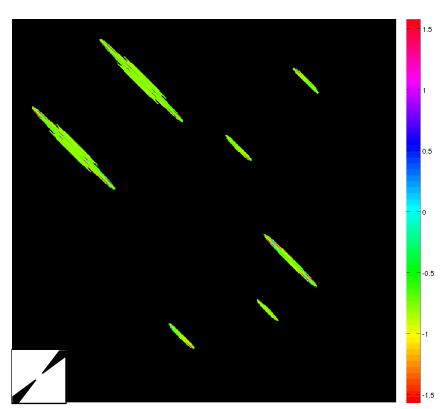

(c) Orientation of the quasimonogenic shearlet coefficients $(T=80)$

Figure 11: Local amplitude, instantaneous phase and local orientation of the quasi-monogenic shearlet coefficients at scale $j=2$ as depicted in Fig. 10 .

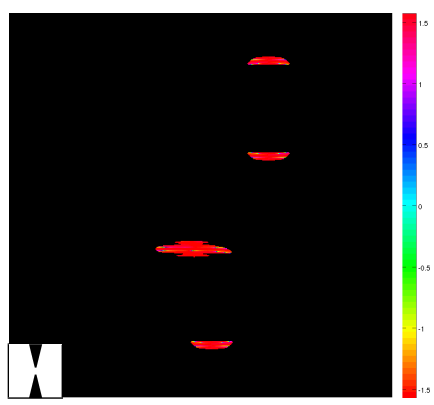

(a) Orientation of the quasimonogenic shearlet coefficients with $j=2$ and $k=0(T=80)$

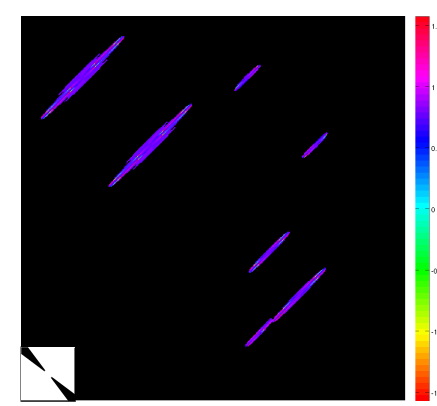

(b) Orientation of the quasimonogenic shearlet coefficients with $j=2$ and $k=4(T=80)$

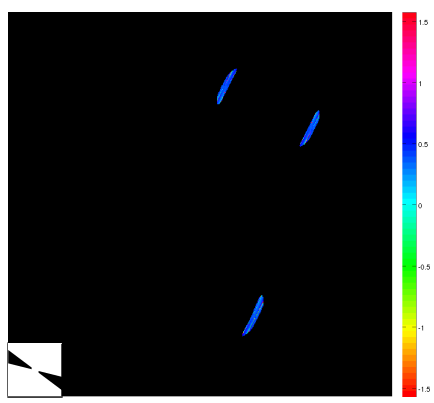

(c) Orientation of the quasimonogenic shearlet coefficients with $j=2$ and $k=2(T=80)$

Figure 12: Orientation of different quasi-monogenic shearlet coefficients 
directional components. Fig. 13(b) shows the amplitude at a coarse scale that corresponds to the scratch in the original image. Fig. 13(c) and 13(d) contain the phase at a higher scale for two different shear parameters which provides a decomposition of the rhombi. The respective orientations are depicted in Fig. 13(e) and 13(f).

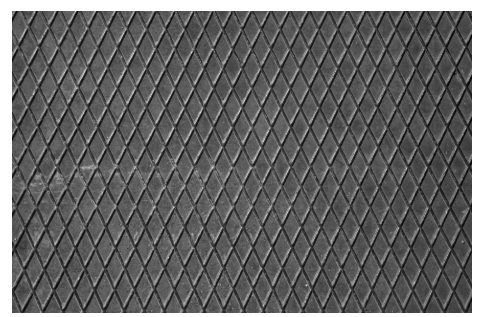

(a) metal plate

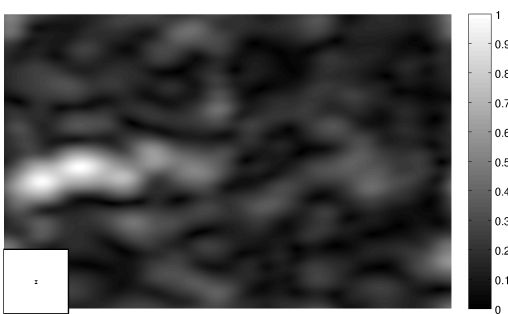

(b) Quasi-monogenic amplitude for $j=$ $0, k=0$ (vertical cone)

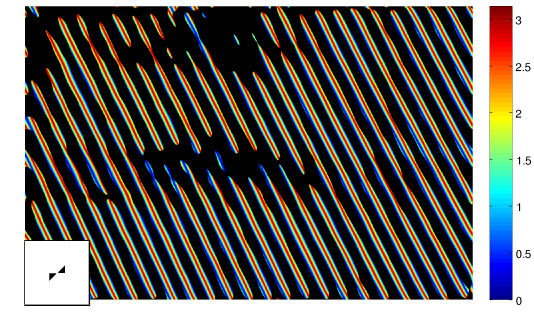

(c) Quasi-monogenic phase for $j=1$, $k=-1$ (horizontal cone)

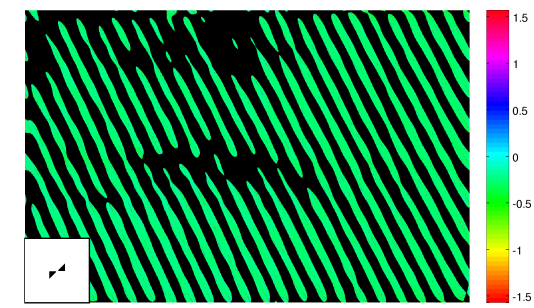

(e) Quasi-monogenic orientation for $j=$ $1, k=-1$ (horizontal cone)

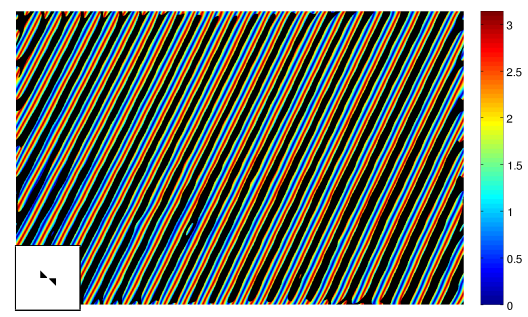

(d) Quasi-monogenic phase for $j=1$, $k=1$ (horizontal cone)

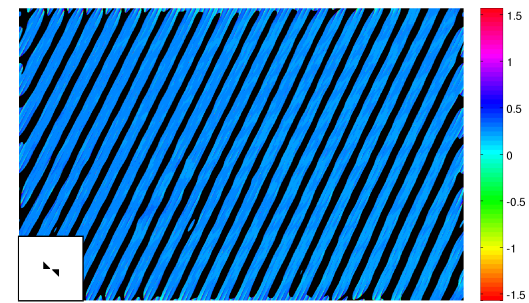

(f) Quasi-monogenic orientation for $j=$ $1, k=1$ (horizontal cone)

Figure 13: Quasi-monogenic amplitude, phase and orientation for a metal plate texture.

In our second experiment we consider the image of the woolen net in Fig. 14(a). The coarse scale amplitude in Fig. 14(b) captures the background of the original image. Fig. 14(c) and Fig. 14(d) show the amplitude and the orientation of the horizontal parts of the net, respectively.

Our last example is the image of a metallic surface with scratches in Fig. 15(a). The illumination can be obtained from the coarse scale amplitude, see Fig. 15(b). Using different shear parameters at a finer scale we can even decompose the scratches. The respective quasimonogenic amplitude, phase and orientation is given in Fig. 15(c)-Fig. 15(h).

\section{Acknowledgements}

The authors wishes to thank J. Ma (Harbin Institute of Technology) for providing Fig. 15(a). G. S. acknowledges the financial support through the DFG Grant STE571/11-1. 


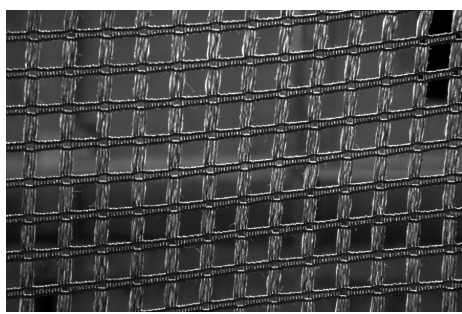

(a) woolen net

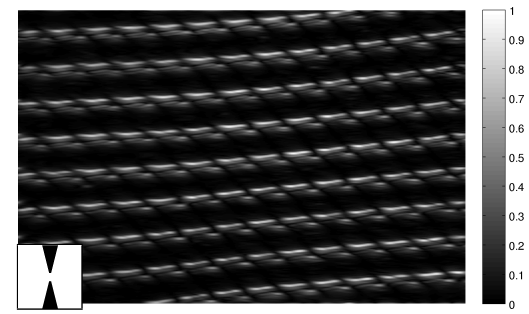

(c) Quasi-monogenic amplitude for $j=$ $2, k=0$ (vertical cone)

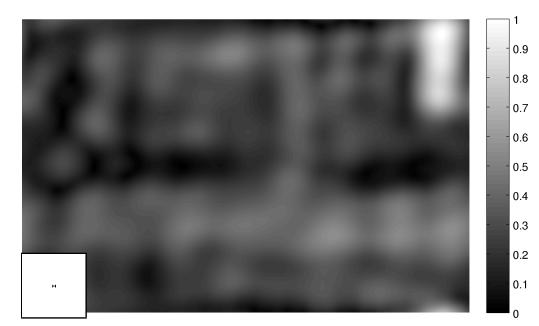

(b) Quasi-monogenic amplitude for $j=$ $0, k=0$ (horizontal cone)

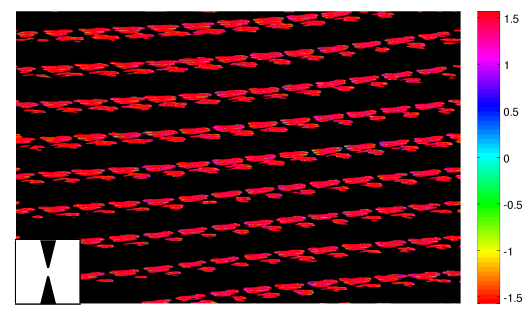

(d) Quasi-monogenic orientation for $j=2, k=0$ (vertical cone)

Figure 14: Quasi-monogenic amplitude, phase and orientation for a woolen net.

B. H. thanks for the support in design of the FPF unit by S. Schausberger at JKU Linz; for the help in design of SLM software by the team of M. Ritsch-Marte at MedUni Innsbruck and by M. Reinhardt at BA Freiberg, for the general support provided by D. Stifter and E.P. Klement at JKU Linz, and B. Heise for discussions. Furthermore, the Federal Ministry of Economy, Family and Youth and the National Foundation for Research, Technology and Development are gratefully acknowledged for funding the Austrian part of work. 


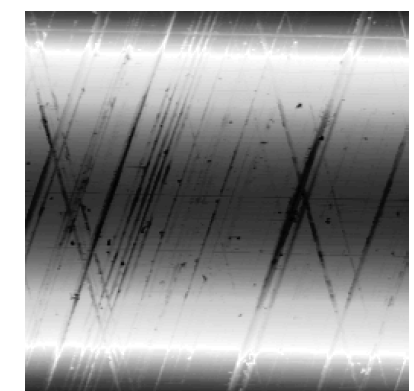

(a) metallic surface with scratches

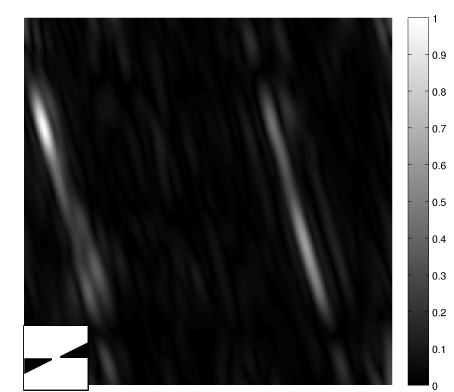

(c) Quasi-monogenic amplitude for $j=2, k=-1$ (horizontal cone)

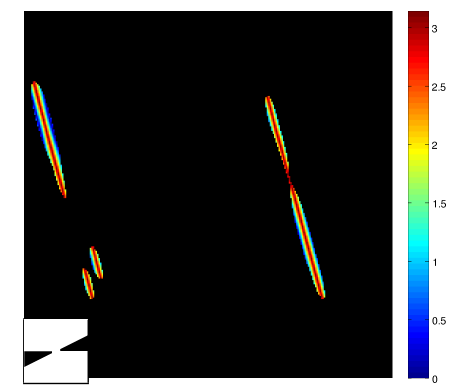

(e) Quasi-monogenic phase for $j=2, k=-1$ (horizontal cone)

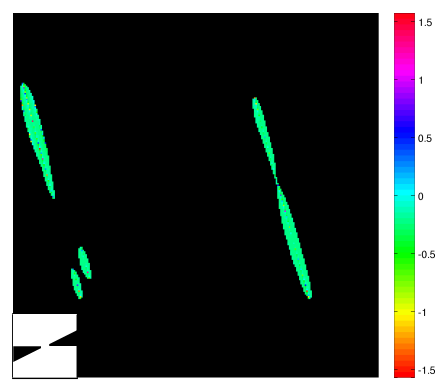

(g) Quasi-monogenic orientation for $j=2, k=-1$ (horizontal cone)

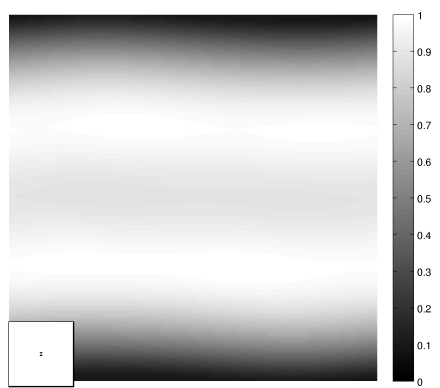

(b) Quasi-monogenic amplitude for $j=0, k=0$ (vertical cone)

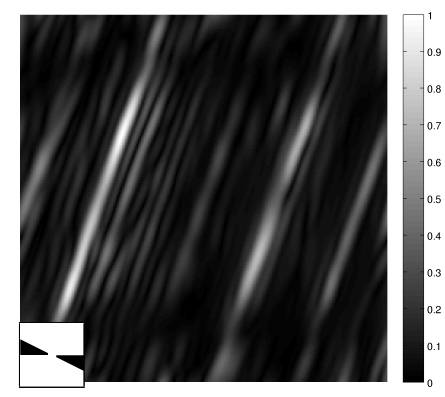

(d) Quasi-monogenic amplitude for $j=2, k=1$ (horizontal cone)

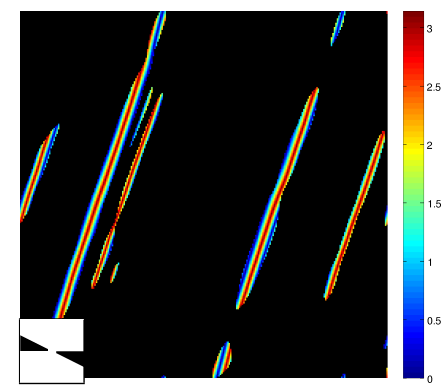

(f) Quasi-monogenic phase for $j=2, k=1$ (horizontal cone)

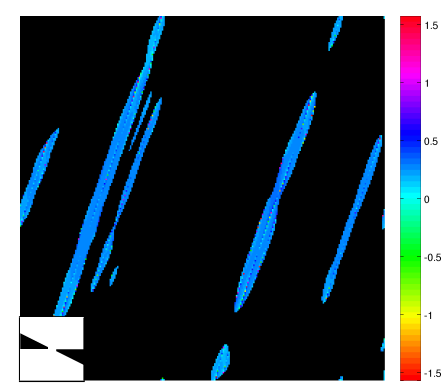

(h) Quasi-monogenic orientation for $j=2, k=1$ (horizontal cone)

Figure 15: Quasi-monogenic amplitude, phase and orientation metallic surface 


\section{References}

[1] S. Bernet, A. Jesacher, M. Fürhapter, C. Maurer, and M. Ritsch-Marte. Quantitative imaging of complex samples by spiral phase contrast microscopy. Optics Express, 14(9): 3792-3805, 2006.

[2] S. Bernstein, J. L. Bouchot, M.Reinhardt, B. Heise. Generalized Analytic Signals in Image Processing: Comparison, Theory and Applications. Quaternion and Clifford Fourier Transforms and Wavelets Trends in Mathematics, pp 221-246, 2013.

[3] E. J. Candes and D. L. Donoho, Curvelets - A surprisingly effective nonadaptive representation for objects with edges, in : Curves and Surfaces, L.L. Schumaker et al., eds, Vanderbild University Press, Nashville, TN (1999).

[4] E. J. Candès, L. Demanet, D. L. Donoho, and L. Ying. Fast discrete curvelet transforms. Multiscale Modeling Simulation, 5(3):861-899, 2006.

[5] S. Dahlke, G. Kutyniok, P. Maass, C. Sagiv, H.-G. Stark, and G. Teschke, The uncertainty principle associated with the continuous shearlet transform, International Journal on Wavelets Multiresolution and Information Processing, 6:157-181, 2008.

[6] S. Dahlke, G. Kutyniok, G. Steidl, and G. Teschke. Shearlet coorbit spaces and associated Banach frames. Applied and Computational Harmonic Analysis, 27(2):195-214, 2009.

[7] M. Felsberg and G. Sommer. The monogenic signal. IEEE Transactions on Signal Processing, 49(12):3136-3144, 2001.

[8] W. Förstner and E. Gülch. A fast operator for detection and precise location of distinct points, corners and centres of circular features. Proc. ISPRS Intercommission Conference on Fast Processing of Photogrammetric Data, Interlaken, Switzerland, pages 281-305, 1987.

[9] J. E. Gilbert and M. Murray. Clifford Algebras and Dirac Operators in Harmonic Analysis. Cambridge University Press, 1991.

[10] J. W. Goodman, Introduction to Fourier Optics. Roberts \& Company Publisher, 2005.

[11] K. Guo, G. Kutyniok, and D. Labate. Sparse multidimensional representations using anisotropic dilation and shear operators. Wavelets und Splines (Athens, GA, 2005), G. Chen und MJ Lai, eds., Nashboro Press, Nashville, TN, pages 189-201, 2006.

[12] K. Guo and D. Labate. Characterization and analysis of edges using the continuous shearlet transform. SIAM Journal on Imaging Science, 2:259-286, 2009.

[13] S. Häuser and G. Steidl. Convex multiclass segmentation with shearlet regularization. International Journal of Computer Mathematics, 90(1):62-81, 2013.

[14] B. Heise, S. Schausberger, D. Stifter. Coherence Probe Microscopy - Contrast Modification and Image Enhancement. Imaging \& Microscopy, 14(1):29-32, 2012.

[15] S. Held, M. Storath, P. Massopust, and B. Forster. Steerable wavelet frames based on the Riesz transform. IEEE Transactions on Image Processing, 19(3):653-67, 2010. 
[16] U. Köthe and M. Felsberg. Riesz-transforms vs. derivatives: On the relationship between the boundary tensor and the energy tensor. In Proceedings of the Scale Space Conference, Springer, pp. 179-191, 2005.

[17] G. Kutyniok and D. Labate. Resolution of the wavefront set using continuous shearlets. Transactions of the American Mathematical Society, 361(5):2719-2754, 2009.

[18] K. G. Larkin, D. J. Bone and M. A. Oldfield. Natural demodulation of two-dimensional fringe patterns. I. General background of the spiral phase quadrature transform. Journal of the Optical Society of America A, 18(8):1862-1870, 2001.

[19] R. S. Laugesen, N. Weaver, G. L. Weiss, and E. N. Wilson. A characterization of the higher dimensional groups associated with continuous wavelets. The Journal of Geometrical Analysis, 12(1):89-102, 2002.

[20] J. Ma and G. Plonka-Hoch. The curvelet transform: A review of recent applications. IEEE Signal Processing Magazine, 27(2):118-133, 2010.

[21] S. Mallat. A Wavelet Tour of Signal Processing: the Sparse Way. Academic Press, San Diego, 2008.

[22] Y. Meyer. Oscillating Patterns in Image Processing and Nonlinear Evolution Equations, AMS, Providence, 2001.

[23] S. E. Schausberger, B. Heise, C. Maurer, S. Bernet, M. Ritsch-Marte, D. Stifter. Flexible contrast for low-coherence interference microscopy by Fourier-plane filtering with a spatial light modulator. Optics Letters, 35(24):4154-4156, 2010.

[24] G. Situ, G. Pedrini, W. Osten. Spiral phase filtering and orientation-selective edge detection/enhancement. JOSA A, 26(8):1788-1797, 2009.

[25] E. M. Stein. Singular Integrals and Differentiability Properties of Functions. Princeton University Press, 1970.

[26] M. Storath. Directional multiscale amplitude and phase decomposition by the monogenic curvelet transform. SIAM Journal on Imaging Science, 4:57-78, 2011.

[27] M. Unser and D. van de Ville. Higher-order Riesz transform and steerable wavelet frames. In Proceedings of the IEEE International Conference on Image Processing, Cairo, pp. 3801-3804, 2009.

[28] M. Unser, D. Sage, and D. van de Ville. Multiresolution monogenic signal analysis using the Riesz-Laplace wavelet transform. IEEE Transactions on Image Processing, 18(11):2402-2418, 2009. 